\title{
Global incidence of Necrotizing Enterocolitis: a systematic review and Meta- analysis
}

Amer Alsaied ${ }^{1,2,3}$, Nazmul Islam ${ }^{1}$ and Lukman Thalib ${ }^{1 *}$ (D)

\begin{abstract}
Background: Necrotizing Enterocolitis (NEC) is a major cause of morbidity and mortality in the Neonatal Intensive Care Unit (NICU), yet the global incidence of NEC has not been systematically evaluated. We conducted a systematic review and meta-analysis of cohort studies reporting the incidence of NEC in infants with Very Low Birth Weight (VLBW).

Methods: The databases searched included PubMed, MEDLINE, the Cochrane Library, EMBASE and grey literature. Eligible studies were cohort or population-based studies of newborns including registry data reporting incidence of NEC. Incidence were pooled using Random Effect Models (REM), in the presence of substantial heterogeneity. Additional, bias adjusted Quality Effect Models (QEM) were used to get sensitivity estimates. Subgroup analysis and meta-regression were used to explore the sources of heterogeneity. Funnel plots as appropriate for ratio measures were used to assess publication bias.
\end{abstract}

Results: A systematic and comprehensive search of databases identified 27 cohort studies reporting the incidence of NEC. The number of neonate included in these studies was 574,692. Of this 39,965 developed NEC. There were substantial heterogeneity between studies $\left(I^{2}=100 \%\right)$. The pooled estimate of NEC based on REM was 7.0\% (95\% Cl: 6.0-8.0\%). QEM based estimate (6.0\%; 95\% Cl: 4.0-9.0\%) were also similar. Funnel plots showed no evidence of publication bias. Although, NEC estimates are similar across various regions, some variation between high and low income countries were noted. Meta regression findings showed a statistically significant increase of NEC over time, quantified by the publication year.

Conclusion: Seven out of 100 of all VLBW infants in NICU are likely to develop NEC. However, there were considerable heterogeneity between studies. High quality studies assessing incidence of NEC along with associated risk factors are warranted.

Keywords: Necrotizing Enterocolitis, Incidence, Systematic review, Meta-analysis

\footnotetext{
* Correspondence: Lthalib@qu.edu.qa

'Department of Public Health, College of Health Sciences, QU Health, Qatar University, Doha, Qatar

Full list of author information is available at the end of the article
}

(c) The Author(s). 2020 Open Access This article is licensed under a Creative Commons Attribution 4.0 International License, which permits use, sharing, adaptation, distribution and reproduction in any medium or format, as long as you give appropriate credit to the original author(s) and the source, provide a link to the Creative Commons licence, and indicate if changes were made. The images or other third party material in this article are included in the article's Creative Commons licence, unless indicated otherwise in a credit line to the material. If material is not included in the article's Creative Commons licence and your intended use is not permitted by statutory regulation or exceeds the permitted use, you will need to obtain permission directly from the copyright holder. To view a copy of this licence, visit http://creativecommons.org/licenses/by/4.0/. The Creative Commons Public Domain Dedication waiver (http://creativecommons.org/publicdomain/zero/1.0/) applies to the data made available in this article, unless otherwise stated in a credit line to the data. 


\section{Background}

Last three decades have witnessed great improvements in the neonatal intensive care, in particular, with the introduction of surfactant therapy and the subsequent improvement in the care of respiratory distress syndrome (RDS) that reduced the mortality among preterm newborns [1]. With better survival of premature babies, Necrotizing Enterocolitis (NEC) became more common and its burden became more prominent [2].

Multiple population-based studies, some based on large cohort studies, have reported the incidence of NEC to vary from 2 to $13 \%$ in preterm and Very Low Birth Weight (VLBW) infants [2-6]. The variation in the incidence were attributed to differences in the risk factor profiles as well as differing population at risk, detection rate and inclusion and exclusion criteria. There is no pooled estimate of the incidence of NEC worldwide. Furthermore, there is no incidence data from some regions such as North Africa, the Middle East or the Arab Gulf region, apart from a single study from the UAE [7].

With the continuing improvement in survival of preterm newborns, the modifiable risk factors of NEC need to be studies and made use of in developing appropriate interventions to reduce the incidence and impact of NEC. In this context, clinicians and researchers have attempted to identify the factors associated with risk and prognosis of NEC. It was reported as early as the 1980's, that there exist an association between rapid advancement of feeding and the onset of NEC [8]. Subsequent reports showed preterm birth $[9,10]$, small birth weight [9-11] and race [11] were also to be important risk factors. Contemporary reports confirm these initial reports and expand the list to include a few more. More recent studies have shown that preterm birth $[3,12]$. low birth weight $[2,12]$, rapid advancement of feeding, race and ethnicity, use of glucocorticosteriods [2], maternal infection [13], indomethacin therapy [14], congenital pneumonia [14], meconium aspiration [15], asphyxia [15], blood transfusion [15] and hypotension within the first week of life [16] are also potential contributing factors.

This study aims to systematically review the incidence reported from different parts of the world to synthesize a global incidence of confirmed NEC in VLBW infants. The study also aims to explore the regional variability as well as other potential factors that can explain variability in the incidence.

\section{Methods}

The recommendations from the Preferred Reporting Items for Systematic Review and Meta-Analysis (PRIS MA) served as the guide in collating and reporting this review [17].

\section{Eligibility criteria}

Eligible studies included cohort or population-based studies of newborns including registry data. Both prospective and retrospective studies were included. Studies reporting the number, frequency or incidence of confirmed NEC in preterm infants or VLBW infants along with appropriate denominator were included. Studies that reported data on subgroups of infants with specific exposures such as congenital heart disease, perinatal infections, preterm rupture of membrane, or sepsis were excluded when the incidence could not be extracted. Studies with unclear case definitions of NEC were also excluded. Randomized controlled trials had strict selection criteria therefore including them would have caused selection bias and reduced the external validity of our pooled estimate. Hence, experimental studies that were assessing the effect of an intervention on a selected group of neonates were excluded. Case series where there were no denominator data to compute the incidence were also excluded.

Incidence is used as opposed to prevalence because of the natural history of NEC and its short duration of disease. It is envisaged that findings form this study would provide clinically important baseline data as the starting point for studies that aim to reduce the incidence of NEC.

\section{Population and outcome}

The VLBW infants formed the population of this study and the outcome of was the incidence of NEC stage II or above according to Bells criteria.

\section{Search data bases}

The database search was started in September 2018 and last updated in December 2019. The databases searched were PUBMED, MEDLINE (Ovid), EMBASE, the Cochrane Library. Additional databases searched included: African Index Medicus Database, Latin America and Caribbean Center of Health Science International, Open Grey, IndMED, KoreaMED, Virtual Health Library, National Library of Australia and Social Care Online.. Further manual search included looking for relevant studies in the reference lists of the included papers.

\section{Search strategy}

The search strategy was developed by the authors to include a comprehensive database search using broader search terms such as: "Enterocolitis, Necrotizing", "Epidemiology", "Incidence", "Cohort Studies", and "population-Based studies", "cohort studies", "epidemiological data", "prematurity", "Very low birth weight", "clinical study", "cohort analysis", and "human". Additional $\mathrm{MeSH}$ (Medical Subject Heading) term based search complemented the above search. When appropriate 
using the above terms with a combination of 'and' and 'or' in accordance with search engine specifications were carried out. The search string used for PUBMED is given in Supplementary file S1 as an illustration.

\section{Study selection}

Two review authors (AA and NI) independently assessed the titles and abstracts of all citations retrieved by the search for relevance against the inclusion criteria. Then the full-text versions of studies considered potentially eligible were retrieved. The same two authors independently assessed the full papers for eligibility, with disagreements resolved through input of the third author. The duplicate records and those not eligible were eliminated and a PRISMA flow chart was created to depict the study selection process.

\section{Data extraction}

Data form the eligible studies were extracted and collated on to data tables. Name of the authors, year of publication, data on the time period covered by the study, location of the study, inclusion and exclusion criteria of the study (Table 1), the reported population at risk and whether it was VLBW infants or preterm infants, case definition, incidence or number on NEC cases and size of population at risk (Table 2) were collected. The data extraction process was performed by AA and checked by NI. Any discrepancies is resolved by discussion.

\section{Risk of Bias assessment}

All the included studies were assessed for internal and external validity using the criteria put forward by Hoy et al. that were specific for prevalence and incidence studies (Fig. 1). This tool was developed based on key domains they identified to be important in assessing the risk of bias in incidence and prevalence studies. The tool was subsequently validated and found to have good validity [30].

\section{Data synthesis}

Pooling the incidence estimates was done after arcsine transformations of the data as it has been shown to stabilize variance and reduce bias [31]. Heterogeneity was assessed using the Cochrane $\mathrm{Q}$ test and Higgin's $\mathrm{I}^{2}$ value. Smaller $p$ values and $\mathrm{I}^{2}>50 \%$ were indicative of significant heterogeneity [32, 33]. As Cochrane guidelines suggest use of Random Effect Models (REM) when significant heterogeneity is encountered [34] we employed REM models estimates to arrive at the main conclusion. Further, bias adjusted Quality Effect Models (QEM) [35] were used to obtain sensitivity estimates to check the robustness of the REM estimates. Quality scores obtained using Hoy's criteria were used in fitting the QEM.

Forest plots were used to display the incidence of NEC with corresponding 95\% confidence intervals. We used Hunter plots to assess the publication bias as Hunter et al. have shown the classical funnel plot to be inappropriate for proportion studies such as prevalence or incidence [36].

A-priori planned meta-regression was performed to evaluate if the publication year has any impact on the variability of the incidence and as a possible cause of heterogeneity. This was also thought to be important to understand if the long term trend in incidence of NEC to see if they are on a rise or decline. Further subgroup analysis by region based on income category of the countries provided by World Bank and population at risk (VLBW or extremely premature) was also carried out [37]. This sub-group analysis was not an a-priori decision but an attempt to explain the variability in NEC due to substantial heterogeneity. Groups consisted of high income countries (HIC) and low middle-income countries (LMIC).

The meta analyses were carried out using MetaXL [31] and the subgroup analysis and meta regression were carried out using Comprehensive Meta-Analysis (CMA-V3) software [38].

\section{Results}

\section{Study characteristics}

The total number of publications identified for screening was 1694. The process of selection of eligible studies are depicted as a PRISMA flow chart (Fig. 2). A total of 27 studies were found to fulfill the eligibility criteria and included in the review (Table 1). The number of neonate included in these studies was 574,692. Of these, 39,965 neonates developed confirmed NEC (Table 2). The studies covered a broader geographical areas globally. Some regions had multiple studies other areas had none. A total of eight studies were reported from the United States covering a number of states including: California, Texas, Atlanta, Connecticut, and New York [3, 6, 9, 18, 19, 39-41]. Multiple studies were also reported from the Europe including Poland, Romania, Finland, Belgium, Sweden and Switzerland [12, 13, 23, 39, 42, 43]. Also, four studies were done in China, Korea, Singapore and Malaysia [14, 16, 44, 45]. Three studies from Australia $[4,21,46]$, one from the Middle East [7] and one from India [24].

The publication year of the studies ranged from 1988 to 2019 , but the majority were carried out after 2000 . Some of the studies focused on evaluating a certain exposure $[7,9,21,43]$, however, the data presented in these papers were not limited to the exposure groups 
Table 1 Characteristics of the included studies

\begin{tabular}{|c|c|c|c|c|c|c|c|}
\hline Author/year & data base studied & Inclusion criteria & Exclusion criteria & $\begin{array}{l}\text { Population at risk } \\
\text { reported }\end{array}$ & NEC case definition & $\begin{array}{l}\text { Comment on } \\
\text { VLBW }\end{array}$ & $\begin{array}{l}\text { Incidence } \\
\text { (cumulative) }\end{array}$ \\
\hline $\begin{array}{l}\text { Stoll et al. } \\
2010 \text { [18] }\end{array}$ & $\mathrm{NICHD}$ & $\begin{array}{l}\text { VLBW infants born } \\
\text { in NRN centers GA } \\
22-28 \text { wks. }\end{array}$ & $\begin{array}{l}\text { Congenital } \\
\text { anomalies }\end{array}$ & $\begin{array}{l}\text { preterm infants } \\
\text { among a VLBW } \\
\text { pool }\end{array}$ & clinically & $\begin{array}{l}\text { exclusively } \\
\text { VLBW infants }\end{array}$ & $11 \%$ \\
\hline $\begin{array}{l}\text { Llanos et al. } \\
2002 \text { [3] }\end{array}$ & $\begin{array}{l}\text { Finger Lakes } \\
\text { regional center }\end{array}$ & $\begin{array}{l}\text { all live births in an } \\
\text { area of } 6 \text { counties. } \\
\text { Data obtained } \\
\text { from a state-wide } \\
\text { registry. }\end{array}$ & not clear & $\begin{array}{l}\text { all newborns in } \\
\text { the regional } \\
\text { center were } \\
\text { accounted for } \\
\text { but specific } \\
\text { report on NEC } \\
\text { stage II and } \\
\text { above among } \\
\text { the VLBW infants } \\
\text { is extracted }\end{array}$ & $\begin{array}{l}\text { NEC stage } \| \text { and } \\
\text { above }\end{array}$ & $\begin{array}{l}\text { population } \\
\text { based study } \\
\text { but reported } \\
\text { specific } \\
\text { parameters on } \\
\text { VLBW }\end{array}$ & $3.29 \%$ \\
\hline $\begin{array}{l}\text { Luig et al. } \\
2005 \text { [4] }\end{array}$ & $\begin{array}{l}\text { New South Wales } \\
\text { - state-wide data } \\
\text { base. NICUS Neo- } \\
\text { natal Intensive } \\
\text { Care Unit Study }\end{array}$ & $\begin{array}{l}\text { population based } \\
\text { study - all preterm } \\
\text { infant s between } \\
24 \text { and } 28 \text { wks. }\end{array}$ & not clear & $\begin{array}{l}\text { all preterm } \\
\text { infants } 24-28 \\
\text { weeks of } \\
\text { gestation }\end{array}$ & $\begin{array}{l}\text { Clinical definition as } \\
\text { confirmed NEC on a } \\
\text { set of criteria similar } \\
\text { to Bell's criteria }\end{array}$ & $\begin{array}{l}\text { the mean birth } \\
\text { weight and SD } \\
\text { of the three } \\
\text { epochs were } \\
959(240), 946 \\
\text { (204), and } 935 \\
(240)\end{array}$ & $7.67 \%$ \\
\hline $\begin{array}{l}\text { Holman } \\
\text { et al. } 2006 \\
{[19]}\end{array}$ & $\begin{array}{l}\text { data from } \\
\text { discharge registry } \\
\text { (the kid's } \\
\text { Inpatient } \\
\text { Database) } \\
\text { compiled data } \\
\text { from } 27 \text { states, } \\
2700 \text { hospitals } \\
\text { accounting for } \\
10 \% \\
\text { uncomplicated } \\
\text { births from these } \\
\text { hospitals }\end{array}$ & $\begin{array}{l}\text { the data is a } \\
\text { comprehensive } \\
\text { cohort of } 10 \% \text { of } \\
\text { all live births in } \\
\text { the specified } \\
\text { hospitals. }\end{array}$ & $\begin{array}{l}\text { NE after } 1 \text { month of } \\
\text { age }\end{array}$ & VLBW infants & $\begin{array}{l}\text { ICD } 9 \text {-CM code NEC } \\
777.5\end{array}$ & $\begin{array}{l}\text { Specific report } \\
\text { NEC and VLBW } \\
\text { infants is } \\
\text { presented } \\
\text { exclusively } \\
\text { VLBW infants }\end{array}$ & $4.34 \%$ \\
\hline $\begin{array}{l}\text { Youn } 2015 \\
{[16]}\end{array}$ & $\begin{array}{l}\text { Korean Neonatal } \\
\text { Network. } \\
\text { Admissions into } \\
55 \text { participating } \\
\text { neonatal intensive } \\
\text { care unites }\end{array}$ & $\begin{array}{l}\text { all live births or } \\
\text { admissions within } \\
28 \text { days. VLBW } \\
\text { infants. Data } \\
\text { collected }\end{array}$ & $\begin{array}{l}52 \text { were diagnosed } \\
\text { with NEC II and } \\
\text { Spontaneous bowel } \\
\text { perforation and were } \\
\text { excluded }\end{array}$ & VLBW infants & $\begin{array}{l}\text { bell's stage II and } \\
\text { above }\end{array}$ & $\begin{array}{l}\text { exclusively } \\
\text { VLBW infants }\end{array}$ & $6.41 \%$ \\
\hline $\begin{array}{l}\text { Qian et al. } \\
2017\end{array}$ & $\begin{array}{l}95 \text { major referral } \\
\text { centers in } 29 \\
\text { provinces. } \\
\text { Representative of } \\
\text { NICU care in the } \\
\text { areas }\end{array}$ & $\begin{array}{l}\text { all LBW infants } \\
\text { were included. }\end{array}$ & not specified & $\begin{array}{l}\text { the study reports } \\
\text { specific } \\
\text { parameters of } \\
\text { VLBW infants }\end{array}$ & $\begin{array}{l}\text { bell's stage II and } \\
\text { above }\end{array}$ & $\begin{array}{l}\text { reports on } \\
\text { VLBW infants } \\
\text { are extracted } \\
\text { from the } \\
\text { publications }\end{array}$ & $2.53 \%$ \\
\hline $\begin{array}{l}\text { Ahle et al. } \\
2013 \text { [12] }\end{array}$ & $\begin{array}{l}\text { Swedish National } \\
\text { Board of Health } \\
\text { and Welfare, the } \\
\text { National Patient } \\
\text { Register, the } \\
\text { Swedish Medical } \\
\text { Birth Register and } \\
\text { the National } \\
\text { Cause of Death } \\
\text { Register }\end{array}$ & $\begin{array}{l}\text { all newborns } \\
\text { between } 1987 \text { and } \\
2009\end{array}$ & $\begin{array}{l}\text { incomplete identity } \\
\text { number }\end{array}$ & VLBW infants & $\begin{array}{l}\text { ICD } 9 \text { or ICD } 10 \\
\text { code } 777 \mathrm{~F} \text { or P77 }\end{array}$ & $\begin{array}{l}\text { reported all } \\
\text { birth weights. } \\
\text { Exact } \\
\text { parameters of } \\
\text { each weights } \\
\text { group are } \\
\text { available too }\end{array}$ & $2.68 \%$ \\
\hline $\begin{array}{l}\text { Wojkowska- } \\
\text { Mach et al. } \\
2014\end{array}$ & $\begin{array}{l}\text { Polish Neonatal } \\
\text { Surveillance } \\
\text { Network }\end{array}$ & $\begin{array}{l}\text { all VLBW infants } \\
\text { born in PNSS }\end{array}$ & missing records & VLBW infants & $\begin{array}{l}\text { NEC defined } \\
\text { according to } \\
\text { Gastmeier's (clinical) }\end{array}$ & $\begin{array}{l}\text { exclusively } \\
\text { VLBW }\end{array}$ & $8.68 \%$ \\
\hline $\begin{array}{l}\text { Boo et al. } \\
2012 \text { [14] }\end{array}$ & $\begin{array}{l}\text { Malaysian } \\
\text { National Neonatal }\end{array}$ & $\begin{array}{l}\text { All VLBW infants in } \\
\text { the MNNR. }\end{array}$ & $\begin{array}{l}\text { excluded infants less } \\
\text { than } 501 \mathrm{~g}\end{array}$ & VLBW infants & $\begin{array}{l}\text { bell's stage II and } \\
\text { above }\end{array}$ & $\begin{array}{l}\text { exclusively } \\
\text { VLBW infants }\end{array}$ & $6.20 \%$ \\
\hline
\end{tabular}


Table 1 Characteristics of the included studies (Continued)

\begin{tabular}{|c|c|c|c|c|c|c|c|}
\hline Author/year & data base studied & Inclusion criteria & Exclusion criteria & $\begin{array}{l}\text { Population at risk } \\
\text { reported }\end{array}$ & NEC case definition & $\begin{array}{l}\text { Comment on } \\
\text { VLBW }\end{array}$ & $\begin{array}{l}\text { Incidence } \\
\text { (cumulative) }\end{array}$ \\
\hline $\begin{array}{l}\text { Wong et al. } \\
2013\end{array}$ & $\begin{array}{l}\text { Population based } \\
\text { study: New South } \\
\text { Wales and } \\
\text { Australian Capital } \\
\text { Territory NICUs } \\
\text { included in the } \\
\text { NICUS }\end{array}$ & $\begin{array}{l}\text { Low birth weight } \\
\text { infants }\end{array}$ & $\begin{array}{l}\text { congenital } \\
\text { malformation, } \\
\text { syndromes with } \\
\text { neurodevelopmental } \\
\text { disorders, death in } \\
\text { the labor room }\end{array}$ & $\begin{array}{l}\text { low birth weights } \\
\text { infants }\end{array}$ & Bell's staging criteria & $\begin{array}{l}\text { the population } \\
\text { was of low } \\
\text { birth weights } \\
\text { (mean birth } \\
\text { weight in two } \\
\text { groups was } \\
895 \text { and } 917 \mathrm{~g} \text {. }\end{array}$ & $7.81 \%$ \\
\hline $\begin{array}{l}\text { Fanaroff } \\
2003[20]\end{array}$ & $\begin{array}{l}\text { NICHD. } \\
\text { Retrospective } \\
\text { data analysis was } \\
\text { performed to } \\
\text { compare three } \\
\text { epochs. }\end{array}$ & Registry data & not specified & VLBW infants & not clear & VLBW infants & $6.23 \%$ \\
\hline $\begin{array}{l}\text { Chedid } \\
\text { et al. } 2008\end{array}$ & $\begin{array}{l}\text { Single large } \\
\text { Neonatal tertiary } \\
\text { referral center }\end{array}$ & $\begin{array}{l}\text { all admission to a } \\
\text { single tertiary } \\
\text { center in Alain } \\
\text { between } 2004 \text { and } \\
2006\end{array}$ & $\begin{array}{l}\text { life threatening } \\
\text { malformation, died in } \\
\text { labor room, less than } \\
500 \mathrm{~g}\end{array}$ & $\begin{array}{l}\text { VLBW infants } \\
\text { (exclude less } \\
\text { than } 500 \mathrm{~g}\end{array}$ & $\begin{array}{l}\text { not clear, } \\
\text { pneumatosis } \\
\text { intestinal or } \\
\text { perforation was used } \\
\text { a confirmation }\end{array}$ & all are VLBW & $5.78 \%$ \\
\hline $\begin{array}{l}\text { Agrawel } \\
\text { et al. } 2015\end{array}$ & $\begin{array}{l}\text { data from single } \\
\text { largest tertiary } \\
\text { hospital in } \\
\text { Singapore. } \\
\text { Viability threshold } \\
\text { less than } 25 \text { wks. } \\
\text { Gestation }\end{array}$ & $\begin{array}{l}\text { Neonates from } \\
\text { High risk VLBW } \\
\text { data base with } \\
\text { GA }<29 \text { wks. }\end{array}$ & $\begin{array}{l}\text { still birth and } \\
\text { miscarriage, less than } \\
23 \text { weeks of } \\
\text { gestation }\end{array}$ & $\begin{array}{l}\text { VLBW and pre- } \\
\text { term }\end{array}$ & $\begin{array}{l}\text { bell's stage II and } \\
\text { above }\end{array}$ & $\begin{array}{l}\text { exclusively } \\
\text { VLBW infants }\end{array}$ & $6.98 \%$ \\
\hline $\begin{array}{l}\text { Patole et al. } \\
2016 \text { [21] }\end{array}$ & $\begin{array}{l}\text { single center } \\
\text { experience. } \\
\text { Comprehensive } \\
\text { retrospective } \\
\text { cohort comparing } \\
\text { a before and after } \\
\text { intervention }\end{array}$ & $\begin{array}{l}\text { all neonates less } \\
\text { than } 34 \text { weeks of } \\
\text { gestation within a } \\
\text { 2-year period be- } \\
\text { fore and after } \\
\text { intervention }\end{array}$ & $\begin{array}{l}\text { neonates involved in } \\
\text { a clinical trial for the } \\
\text { same purpose }\end{array}$ & $\begin{array}{l}\text { the study } \\
\text { reported all } \\
\text { neonates less } \\
\text { than } 34 \text { wks. But } \\
\text { data on }<28 \\
\text { weeks and epoch } \\
1 \text { were extracted }\end{array}$ & $\begin{array}{l}\text { bell's stage II and } \\
\text { above }\end{array}$ & $\begin{array}{l}\text { the birth } \\
\text { weight of the } \\
\text { preterm babies } \\
\text { was not } \\
\text { specifically } \\
\text { reported }\end{array}$ & $6.40 \%$ \\
\hline $\begin{array}{l}\text { Verstreate } \\
\text { et al. } 2016\end{array}$ & $\begin{array}{l}\text { Retrospective } \\
\text { cohort study from } \\
\text { a single e center } \\
\text { using a local audit } \\
\text { data base }\end{array}$ & $\begin{array}{l}\text { All neonates in } \\
\text { the hospital } \\
\text { system }\end{array}$ & $\begin{array}{l}\text { neonates with } \\
\text { culture samples that } \\
\text { had probably } \\
\text { contamination }\end{array}$ & $\begin{array}{l}\text { data on VLBW } \\
\text { was extracted } \\
\text { only }\end{array}$ & clinical definition & $\begin{array}{l}\text { the data } \\
\text { extracted } \\
\text { represents } \\
\text { exclusively } \\
\text { VLBW infants }\end{array}$ & $16.23 \%$ \\
\hline $\begin{array}{l}\text { Harkin et al. } \\
2017\end{array}$ & $\begin{array}{l}\text { Finish Medical } \\
\text { Birth Register } \\
\text { (preterm < } 32 \\
\text { wks.) 22-31. all } \\
\text { VLGA } 4143\end{array}$ & $\begin{array}{l}\text { all born less than } \\
32 \text { weeks of } \\
\text { gestation }\end{array}$ & $\begin{array}{l}\text { congenital } \\
\text { malformations sever } \\
\text { chromosomal defects } \\
\text { or death before } 7 \\
\text { days od life }\end{array}$ & $\begin{array}{l}\text { less than } 28 \\
\text { weeks of } \\
\text { gestation }\end{array}$ & clinical criteria & $\begin{array}{l}50 \% \text { less than } \\
1000 \mathrm{~g} \text { in the } \\
\text { entire } \\
\text { populations. } \\
\text { But weight of } \\
\text { the }<28 \text { weeks } \\
\text { of gestation } \\
\text { was not } \\
\text { specified }\end{array}$ & $6.58 \%$ \\
\hline $\begin{array}{l}\text { Andersen } \\
\text { et al. } 2018\end{array}$ & $\begin{array}{l}\text { birth cohort of } \\
\text { the California } \\
\text { Office Statewide } \\
\text { Health and } \\
\text { Development } \\
\text { (OSHPD) }\end{array}$ & $\begin{array}{l}\text { all live births with } \\
\text { GA 22-36 }\end{array}$ & $\begin{array}{l}\text { chromosomal } \\
\text { abnormalities }\end{array}$ & $\begin{array}{l}\text { GA less than } 28 \\
\text { weeks }\end{array}$ & ICD-9 & $\begin{array}{l}\text { no clear } \\
\text { specification of } \\
\text { the birth } \\
\text { weight of the } \\
\text { preterm } \\
\text { subpopulation }\end{array}$ & $9.10 \%$ \\
\hline $\begin{array}{l}\text { Suciu et al. } \\
2017 \text { [22] }\end{array}$ & $\begin{array}{l}\text { From three } \\
\text { Romanian } \\
\text { hospitals (tertiary } \\
\text { centers) data from } \\
\text { two different } \\
\text { periods 2007- } \\
2010 \text { and 2011-- } \\
2014\end{array}$ & $\begin{array}{l}\text { all preterm babies } \\
\text { less than } 28 \text { weeks } \\
\text { of gestation }\end{array}$ & $\begin{array}{l}\text { chromosomal } \\
\text { abnormalities and } \\
\text { birth defects or } \\
\text { missing data }\end{array}$ & $\begin{array}{l}\text { preterm babies } \\
\text { less than } 28 \\
\text { weeks of } \\
\text { gestation }\end{array}$ & $\begin{array}{l}\text { bell's stage II and } \\
\text { above }\end{array}$ & $\begin{array}{l}\text { the mean birth } \\
\text { and SD of the } \\
\text { two epochs } \\
\text { were } 809+/- \\
211 \text { and } 958 \\
+/-149\end{array}$ & $17.08 \%$ \\
\hline $\begin{array}{l}\text { Patel et al. } \\
2016\end{array}$ & $\begin{array}{l}\text { Prospective } \\
\text { Observational }\end{array}$ & VLBW infants & not specified & VLBW infants & $\begin{array}{l}\text { bell's stage II and } \\
\text { above. Cumulative }\end{array}$ & $\begin{array}{l}\text { exclusively } \\
\text { VLBW infants }\end{array}$ & $7.34 \%$ \\
\hline
\end{tabular}


Table 1 Characteristics of the included studies (Continued)

\begin{tabular}{|c|c|c|c|c|c|c|c|}
\hline Author/year & data base studied & Inclusion criteria & Exclusion criteria & $\begin{array}{l}\text { Population at risk } \\
\text { reported }\end{array}$ & NEC case definition & $\begin{array}{l}\text { Comment on } \\
\text { VLBW }\end{array}$ & $\begin{array}{l}\text { Incidence } \\
\text { (cumulative) }\end{array}$ \\
\hline & $\begin{array}{l}\text { multicenter birth } \\
\text { cohort study } \\
\text { evaluating VLBW } \\
\text { infants from } \\
\text { multiple Level III } \\
\text { neonatal centers } \\
\text { for exposure } \\
\text { blood transfusion } \\
\text { (a risk of NEC) }\end{array}$ & & & & incidence at 8 weeks & & \\
\hline $\begin{array}{l}\text { Bajwa et al. } \\
2011 \text { [23] }\end{array}$ & $\begin{array}{l}\text { Swiss Neonatal } \\
\text { Network. Double } \\
\text { verification by the } \\
\text { Swiss Society of } \\
\text { Neonatology. }\end{array}$ & $\begin{array}{l}\text { The data set } \\
\text { includes all infants } \\
<32 \text { weeks of } \\
\text { gestation } \\
\text { and }>23 \text { wks. }\end{array}$ & $\begin{array}{l}\text { infants who died in } \\
\text { labor room }\end{array}$ & $\begin{array}{l}\text { preterm less than } \\
28 \text { weeks of } \\
\text { gestation }\end{array}$ & clinical definition & $\begin{array}{l}\text { no comment } \\
\text { on the birth } \\
\text { weight of the } \\
\text { subpopulation } \\
\text { less than } 28 \\
\text { weeks of } \\
\text { gestation }\end{array}$ & $4.95 \%$ \\
\hline $\begin{array}{l}\text { Narang } \\
\text { et al. } 1993 \\
\text { [24] }\end{array}$ & $\begin{array}{l}\text { Single Neonatal } \\
\text { Intensive Care } \\
\text { Unit }\end{array}$ & $\begin{array}{l}\text { All live births } \\
\text { during the period } \\
\text { January } 1986 \text { to } \\
\text { September } 1990\end{array}$ & Not reported & $\begin{array}{l}\text { VLBW infants and } \\
\text { pretenn infants } \\
\text { of gestational } \\
\text { age less than } 32 \\
\text { weeks }\end{array}$ & $\begin{array}{l}\text { modified Bell's } \\
\text { criteria }\end{array}$ & $\begin{array}{l}\text { Majority are } \\
\text { VLBW infants }\end{array}$ & $1.5 \%$ \\
\hline $\begin{array}{l}\text { Lodha } 2019 \\
\text { [25] }\end{array}$ & $\begin{array}{l}\text { Tertiary neonatal } \\
\text { intensive care } \\
\text { units participating } \\
\text { in the Canadian } \\
\text { Neonatal Network }\end{array}$ & $\begin{array}{l}\text { born at } 22 \text { to } 28 \\
\text { weeks' gestational } \\
\text { age }\end{array}$ & $\begin{array}{l}\text { birth outside a } \\
\text { tertiary-level NICU, } \\
\text { moribund at birth, } \\
\text { designated as need- } \\
\text { ing palliative care be- } \\
\text { fore delivery, had } \\
\text { major congenital } \\
\text { anomalies, or lacked } \\
\text { cord clamping } \\
\text { information }\end{array}$ & $\begin{array}{l}22 \text { to } 28 \text { weeks' } \\
\text { gestational age }\end{array}$ & $\begin{array}{l}\text { According to the } \\
\text { modified Bell criteria, } \\
\text { and NEC stage } 2 \text { or } \\
\text { higher was classified } \\
\text { as medical or } \\
\text { surgical. }\end{array}$ & $\begin{array}{l}\text { No estimate of } \\
\text { the } \\
\text { percentage of } \\
\text { VLBW infants }\end{array}$ & $9 \%$ \\
\hline $\begin{array}{l}\text { Boghossian } \\
2018[26]\end{array}$ & $\begin{array}{l}\text { Vermont Oxford } \\
\text { Network center }\end{array}$ & $\begin{array}{l}\text { Inborn, singleton } \\
\text { infants without } \\
\text { congenital } \\
\text { malformations }\end{array}$ & $\begin{array}{l}\text { Infants with } \\
\text { unknown sex and } \\
\text { missing or } \\
\text { implausible birth } \\
\text { weight }\end{array}$ & $\begin{array}{l}\text { Infants of } \\
\text { gestational ages } \\
22 \text { to } 29 \text { weeks }\end{array}$ & $\begin{array}{l}\text { diagnosed at surgery } \\
\text { or postmortem or } \\
\text { required at least } 1 \\
\text { clinical sign (eg, } \\
\text { bilious gastric } \\
\text { aspirate, abdominal } \\
\text { distension, or occult } \\
\text { blood in stool) and } \\
\text { at least } 1 \\
\text { radiographic finding } \\
\text { (eg, pneumatosis } \\
\text { intestinalis, } \\
\text { hepatobiliary gas, or } \\
\text { pneumoperitoneum). }\end{array}$ & $\begin{array}{l}\text { the mean birth } \\
\text { weight and SD } \\
\text { of the each } \\
\text { weeks } \\
\text { reported. }\end{array}$ & $9 \%$ \\
\hline $\begin{array}{l}\text { Persson } \\
2018[27]\end{array}$ & $\begin{array}{l}7 \text { national } \\
\text { networks in high- } \\
\text { income countries } \\
\text { that are part of } \\
\text { the International } \\
\text { Neonatal Network } \\
\text { for Evaluating } \\
\text { Outcomes in } \\
\text { Neonates }\end{array}$ & $\begin{array}{l}\text { All singleton } \\
\text { infants born alive } \\
\text { in high-income } \\
\text { countries who } \\
\text { were very preterm } \\
\text { ( } 24-31 \text { weeks' ges- } \\
\text { tation) and with a } \\
\text { birth weight of } \\
\text { less than } 1500 \mathrm{~g}\end{array}$ & $\begin{array}{l}\text { Multiple pregnancies } \\
\text { and major congenital } \\
\text { malformations }\end{array}$ & $\begin{array}{l}\text { Very Preterm and } \\
\text { Very Low-Birth- } \\
\text { Weight Infants }\end{array}$ & $\begin{array}{l}\text { Necrotizing } \\
\text { enterocolitis was } \\
\text { analyzed in a } \\
\text { subgroup of the } \\
\text { cohort because data } \\
\text { from the UKNC were } \\
\text { not available for } \\
\text { stage } 2 \text { or } 3 \text { NEC }\end{array}$ & $\begin{array}{l}\text { Very Preterm } \\
\text { and Very Low- } \\
\text { Birth-Weight } \\
\text { Infants }\end{array}$ & $3 \%$ \\
\hline $\begin{array}{l}\text { Suzuki } 2018 \\
\text { [28] }\end{array}$ & $\begin{array}{l}\text { Neonatal } \\
\text { Research Network }\end{array}$ & $\begin{array}{l}\text { Extremly preterm } \\
\text { infants born } \\
\text { between } 2008 \text { and } \\
2012\end{array}$ & $\begin{array}{l}\text { Infants who died } \\
\text { within } 6 \text { days, infants } \\
\text { with congenital } \\
\text { anomalies, whose } \\
\text { sex was } \\
\text { undetermined, or } \\
\text { whose records were } \\
\text { missing data }\end{array}$ & $\begin{array}{l}\text { extremely } \\
\text { preterm infants }\end{array}$ & $\begin{array}{l}\text { NEC was defined as } \\
\text { stage II/III cases, } \\
\text { according to the } \\
\text { classifications of Bell }\end{array}$ & $\begin{array}{l}\text { All are VLBW } \\
\text { with extremly } \\
\text { preterm }\end{array}$ & $4 \%$ \\
\hline Boghossian & 852 US centers & Infants born & Multiples and infants & Large for & NEC was diagnosed & Mean and SD & $7 \%$ \\
\hline
\end{tabular}


Table 1 Characteristics of the included studies (Continued)

\begin{tabular}{|c|c|c|c|c|c|c|c|}
\hline Author/year & data base studied & Inclusion criteria & Exclusion criteria & $\begin{array}{l}\text { Population at risk } \\
\text { reported }\end{array}$ & NEC case definition & $\begin{array}{l}\text { Comment on } \\
\text { VLBW }\end{array}$ & $\begin{array}{l}\text { Incidence } \\
\text { (cumulative) }\end{array}$ \\
\hline 2018 [29] & $\begin{array}{l}\text { participating in } \\
\text { the Vermont } \\
\text { Oxford Network }\end{array}$ & $\begin{array}{l}\text { between } 154 \text { days } \\
\text { ( } 22 \text { weeks and } 0 \\
\text { days) and } 209 \\
\text { days ( } 29 \text { weeks } \\
\text { and } 6 \text { days) of } \\
\text { gestation }\end{array}$ & $\begin{array}{l}\text { born with congenital } \\
\text { malformations }\end{array}$ & $\begin{array}{l}\text { Gestational Age } \\
\text { Infants }\end{array}$ & $\begin{array}{l}\text { at surgery or } \\
\text { postmortem or } \\
\text { required at least } 1 \\
\text { clinical sign (eg, } \\
\text { bilious gastric } \\
\text { aspirate, abdominal } \\
\text { distension, occult } \\
\text { blood in stool) and } \\
\text { at least } 1 \\
\text { radiographic finding } \\
\text { (eg, pneumatosis } \\
\text { intestinalis, } \\
\text { hepatobiliary gas, or } \\
\text { pneumoperitoneum) }\end{array}$ & $\begin{array}{l}\text { birth weights } \\
\text { reported }\end{array}$ & \\
\hline $\begin{array}{l}\text { Beltempo } \\
2018\end{array}$ & $\begin{array}{l}\text { Canadian } \\
\text { Neonatal Network }\end{array}$ & $\begin{array}{l}\text { Infants born from } \\
22 \text { to } 28 \text { weeks' } \\
\text { GA and admitted } \\
\text { to } 30 \text { Level } 3 \\
\text { neonatal intensive } \\
\text { care units (NICUs) }\end{array}$ & $\begin{array}{l}\text { Infants moribund on } \\
\text { admission or where } \\
\text { palliative care was } \\
\text { provided at birth due } \\
\text { to imminent } \\
\text { mortality, infants } \\
\text { with major } \\
\text { congenital } \\
\text { anomalies, and } \\
\text { infants with missing } \\
\text { SNAP-\|l }\end{array}$ & $\begin{array}{l}\text { Extremely } \\
\text { preterm infants }\end{array}$ & $\begin{array}{l}\text { NEC is defined as } \\
\text { stage } \geq 2 \text { according } \\
\text { to Bell's criteria }\end{array}$ & $\begin{array}{l}\text { Mean and SD } \\
\text { birth weights } \\
\text { of both cohort } \\
\text { is reported }\end{array}$ & $8 \%$ \\
\hline
\end{tabular}

and data from the general population was extracted to compute the incidence (Table 2).

\section{Qualitative review}

Andersone et al. reviewed a cohort data from the California Office Statewide Health Planning And Development [OS HPD] [39]. Upon retrograde calculation of the number of NEC cases and dividing them by a total number of NICU preterm babies the incidence of NEC was 9.1\%. Whilst, Patole et al. conducted a retrospective cohort study reviewing 1755 neonates who were less than 34 weeks of gestation [21]. The aim was to study the effect on the incidence of NEC. In the control group (prior to the initiation of probiotic), there were 835 babies. Among those 250 were preterm with gestational age less than 28 weeks. Stage II or above NEC was found in 16 cases (6\% of preterm controls).

Stoll et al. [38] analyzed data on 9575 newborns with very low birthweight and extremely low gestational age. The incidence in this population was $11 \%$. Llanos et al. [3] reported the incidence among VLBW infants therefore was $3.29 \%$. They used a retrospectively conducted a population-based survey from six counties in New York State. Holeman et al. analyzed the hospital discharge data from the Kid's Inpatient Database from the year 2000 [47]. Among those born with weight less than $1500 \mathrm{~g}$, the number of cases was 2554 and the rate was
4342.8 per 100,000 live births annually with an incidence of $4.3 \%$. Fanaroff et al. evaluated VLBW infants and compared three periods of time: 1987-1988, 1993-1994, and 1999-2000 [20]. The analysis aimed to compare the outcome across the time periods. They showed that the incidence of NEC did not change over time.

Bajwa et al. reviewed the data from the Swiss neonatal network that conatins comprehensive population-based data of all infants in Switzerland [23]. The analysis included 368,055 infants born between 2000 and 2004, Ahle et al. collected data from the Swedish National Board of Health and Welfare, the National Patient Register, the Swedish Medical Birth Register and The National Cause of Death Register between 1987 and 2009 [12]. The incidence of NEC in less than 750 g, 750-999 g, $1000-1499 \mathrm{~g}$ and $1500-2499 \mathrm{~g}$ were $5.31,4.16,1.52$, and $0.007 \%$, respectively.

Verstrate et al. based on a retrospective cohort of 5134 neonatal intensive care unit admissions from a single hospital Belgium found 973 cases were born with a very low birthweight of less than $1500 \mathrm{~g}$ [42]. The incidence of NEC with stage II or above, in this subgroup was $16.23 \%$. Härkin et al. reviewed the data from the national Registry of preterm infants born between 2005 and 2013 in Finland [43]. The incidence of NEC among preterm babies was therefore $16.58 \%$. Wójkowska-Mach et al. reviewed the Polish Neonatal Surveillance Network for 
Table 2 Summary of the 27 studies included in the quantitative analysis

\begin{tabular}{|c|c|c|c|c|c|c|}
\hline Period & Author/Year & Location & Population at risk & $\begin{array}{l}\text { Cases of NEC in population at } \\
\text { risk }\end{array}$ & $\begin{array}{l}\text { Population at } \\
\text { risk }\end{array}$ & Incidence \\
\hline 2003-2007 & Stoll et al. 2010 [18] & US & VLBW infants & not reported $^{a}$ & 9575 & $11.0 \%$ \\
\hline 1991-1998 & Llanos et al. 2002 [3] & US & VLBW infants & 47 & 1425 & $3.29 \%$ \\
\hline $\begin{array}{l}86 / 87,92 / 93, \text { and } 98 / \\
99\end{array}$ & Luig et al. 2005 [4] & Australia & Extremely premature & 127 & 1655 & $7.67 \%$ \\
\hline 2000 & Holman et al. 2006 [19] & $\begin{array}{l}\text { US- } 27 \\
\text { states }\end{array}$ & VLBW infants & 2554 & 58,810 & $4.34 \%$ \\
\hline $201-2014$ & Youn 2015 [16] & Korea & VLBW infants & 149 & 2326 & $6.41 \%$ \\
\hline 2011 & Qian et al. 2017 & China & VLBW infants & 221 & 8727 & $2.53 \%$ \\
\hline 1987-2009 & Ahle et al. 2013 [12] & Sweden & VLBW infants & 473 & 17,608 & $2.68 \%$ \\
\hline 2009 & $\begin{array}{l}\text { Wojkowska-Mach et al. } \\
2014\end{array}$ & Poland & VLBW infants & 79 & 910 & $8.68 \%$ \\
\hline 2007 & Boo et al. 2012 [14] & Malaysia & VLBW infants & 222 & 3601 & $6.20 \%$ \\
\hline 1998-2004 & Wong et al. 2013 & Australia & VLBW infants & 199 & 2549 & $7.81 \%$ \\
\hline 87/88, 93/94,99/2000 & Fanaroff 2003 [20] & US & VLBW infants & 786 & 12,628 & $6.23 \%$ \\
\hline 2004-2006 & Chedid et al. 2008 & UAE & VLBW infants & 10 & 173 & $5.78 \%$ \\
\hline 2000-20,209 & Agrawel et al. 2015 & Singapore & VLBW infants & 50 & 835 & $6.98 \%$ \\
\hline 2008-2010 & Patole et al. 2016 [21] & Australia & Extremely premature & 16 & 250 & $6.40 \%$ \\
\hline 2002-2011 & Verstreate et al. 2016 & Belgium & VLBW infants & 158 & 973 & $16.23 \%$ \\
\hline 2005-2013 & Harkin et al. 2017 & Finland & Extremely premature & 170 & 1025 & $6.58 \%$ \\
\hline 2007-2012 & Andersen et al. 2018 & $\begin{array}{l}\text { US- } \\
\text { California }\end{array}$ & Extremely premature & 1360 & 14,941 & $9.10 \%$ \\
\hline 2007-2010 & Suciu et al. 2017 [22] & Romania & Extremely premature & 82 & 480 & $17.08 \%$ \\
\hline 2010-2014 & Patel et al. 2016 & US-Atlanta & VLBW infants & 44 & 598 & $7.34 \%$ \\
\hline 2000-2004 & Bajwa et al. 2011 [23] & Switzerland & Extremely premature & 64 & 1283 & $4.95 \%$ \\
\hline 1986-1990 & Narang et al. 1993 [24] & India & VLBW infants & & & \\
\hline 2011-2015 & Lodha 2019 [25] & Canada & Extremely premature & 412 & 4680 & $9 \%$ \\
\hline 2006-2016 & Boghossian 2018 [26] & $\begin{array}{l}\text { United } \\
\text { States }\end{array}$ & $\begin{array}{l}\text { VLBW and Extremely } \\
\text { premature }\end{array}$ & 18,129 & 194,736 & $9 \%$ \\
\hline 2007-2015 & Persson 2018 [27] & Sweden & Extremely premature & 2077 & 76,360 & $3 \%$ \\
\hline 2008-2012 & Suzuki 2018 [28] & Japan & Extremely premature & 296 & 8245 & $4 \%$ \\
\hline 2006-2014 & Boghossian 2018 [29] & USA & Extremely premature & 10,376 & 138,869 & $7 \%$ \\
\hline 2010-2015 & Beltempo 2018 & Canada & Extremely premature & 778 & 9230 & $8 \%$ \\
\hline
\end{tabular}

${ }^{a}$ The number of NEC cases was calculated from the incidence and the baseline population for this study

1. Was the study's target population a close representation of the national population in relation to relevant variables?

2. Was the sampling frame a true or close representation of the target population?

3. Was some form of random selection used to select the sample, OR was a census undertaken?

4. Was the likelihood of nonresponse bias minimal?

5. Were data collected directly from the subjects (as opposed to a proxy)?

6. Was an acceptable case definition used in the study?

7. Was the study instrument that measured the parameter of interest shown to have validity and reliability?

8. Was the same mode of data collection used for all subjects?

9. Was the length of the shortest prevalence period for the parameter of interest appropriate?

10. Were the numerator(s) and denominator(s) for the parameter of interest appropriate?

Fig. 1 The 10 criteria used to assess the risk of bias in each included studies 


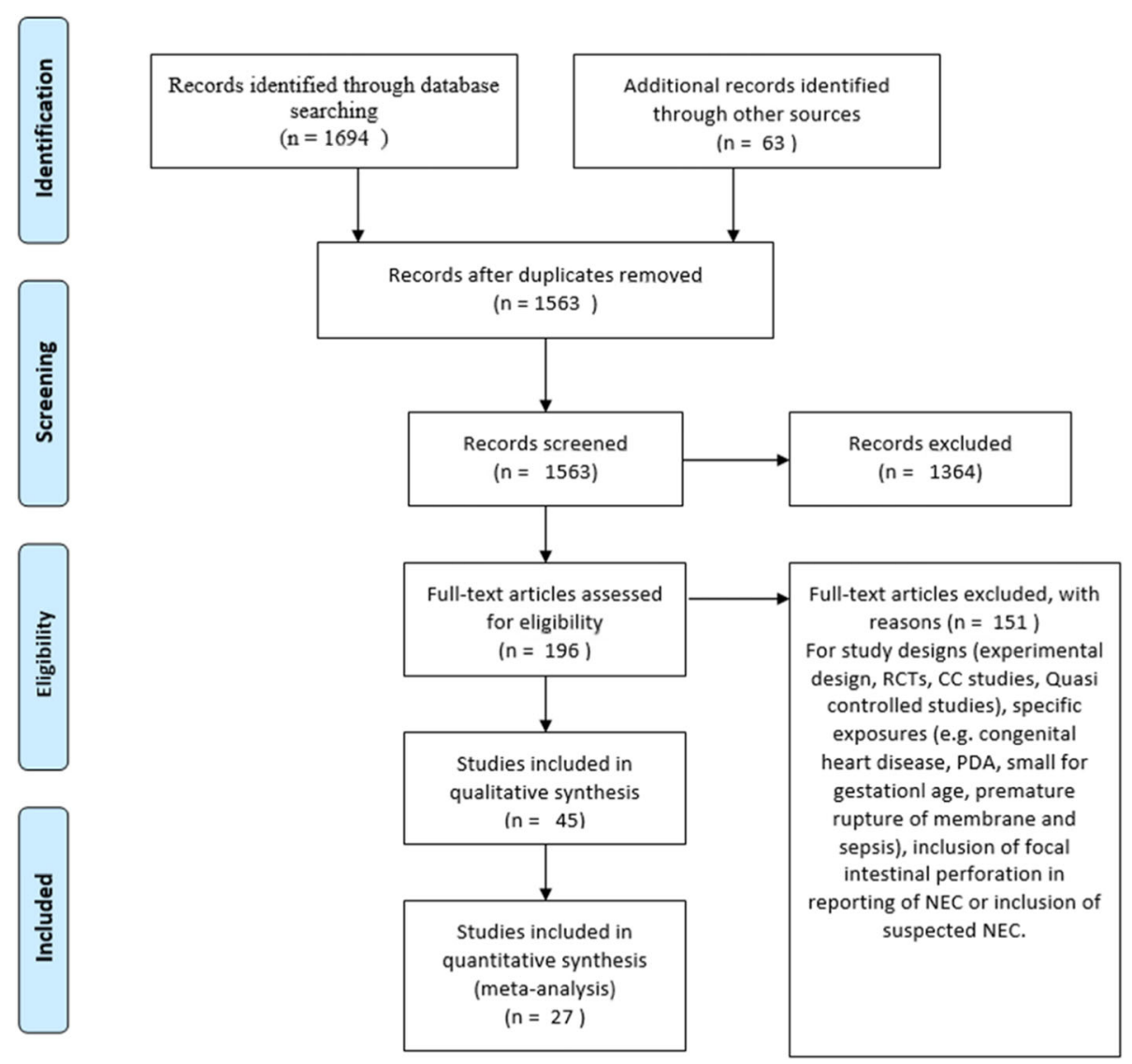

Fig. 2 Flow chart depicting the studies screened, selected and included based on PRISMA

all VLBW infants recorded in the national registry. They used clinical criteria for the definition of NEC and 79 of 910 babies developed NEC [13].

Suciu et al. reviewed data from three tertiary centers in Romania. The study included 480 preterm babies born before 28 weeks of gestation [22]. The incidence was estimated to be $16.6 \%$. The Bell's criteria were used to define cauterizing enterocolitis as stage II and above in this study. Agarwal et al. collected data from the single largest neonatal center in Singapore with a vitality threshold defined at 25 weeks of gestation [45]. The database included all neonates who are with VLBW and gestational age less than 29 weeks. Bell's classification was used to define NEC. 50 babies among 835 developed NEC.

Qian et al. reported data extracted retrospectively from 95 major referral centers and hospitals in china covering a large area of 29 provinces [44]. VLBW infants were specified and the incidence of NEC according to Bell's criteria was presented in 2011 . The data included 46,686 infants of whom, 8727 were born with VLBW. The incidence of confirmed NEC in VLBW infants was 6.5 among a cohort of 8727 infants.

Youn et al. reported a large cohort from South Korea. Among a total of 2326 infant with VLBW, 145 (6.8\%) were diagnosed with confirmed NEC stage II of above [16]. Boo et al. collected data retrospectively from 31 neonatal intensive care units around Malaysia on NEC defined by Bell's criteria among VLBW infants. Among the 3601 babies included, 222 developed NEC. Of these 197 had NEC II and 25 were NEC III or above according to Bell's staging criteria. The incidence was $6.2 \%$ [14]. Luig et al. reported data on all infants born between 24 to 28 weeks of gestation in New South Wales and England, over three different time periods: 1986-1987, 1992-1993, and 1998-1999 [4]. The population included 1655 cases from the three groups divided to 360,622 , and 673 cases in time periods 1986-1987, 1992-1993, and 1998-1999 respectively. Over the entire population the incidence was $7.67 \%$.

Wong et al. conducted a retrospective cohort study reviewing 2549 neonates from 10 neonatal intensive care 
units serving New South Wales in Australia [46]. This study population accounted for all preterm infants in the region of Australia between 1998 and 2004. The conducted the analysis complaining those exposed to steroids and those who were not. The incidence of NEC was $7.8 \%$ as 199 cases developed necrotizing enterocolitis among 2549 preterm babies born before 29 weeks of gestation.

Narang et al. 1993, collected 2200 admissions to the NICU during the period January 1986to September 1990 [24]. Among them 33 developed NEC (Bell's stage $\geq 2$ ). The incidence was $1.5 \%$. Chedid et al. reviewed 173 newborns from 1 Tertiary Referral Center in UAE, Al Ain. All the cohort were born with weight less than $1500 \mathrm{~g}$ [very low birthweight infants] [7]. NEC was diagnosed clinically. Among the study population, 10 babies developed confirmed NEC. The incidence of NEC was $5.8 \%$.

Lodha et al. 2019, compared neonatal outcomes after deferred cord clamping and immediate cord clamping in extremely low-gestational-age neonates from tertiary neonatal intensive care units participating in theestimated incidence based on Canadian Neonatal Network in 2019 was $9 \%$ (43)9\%.

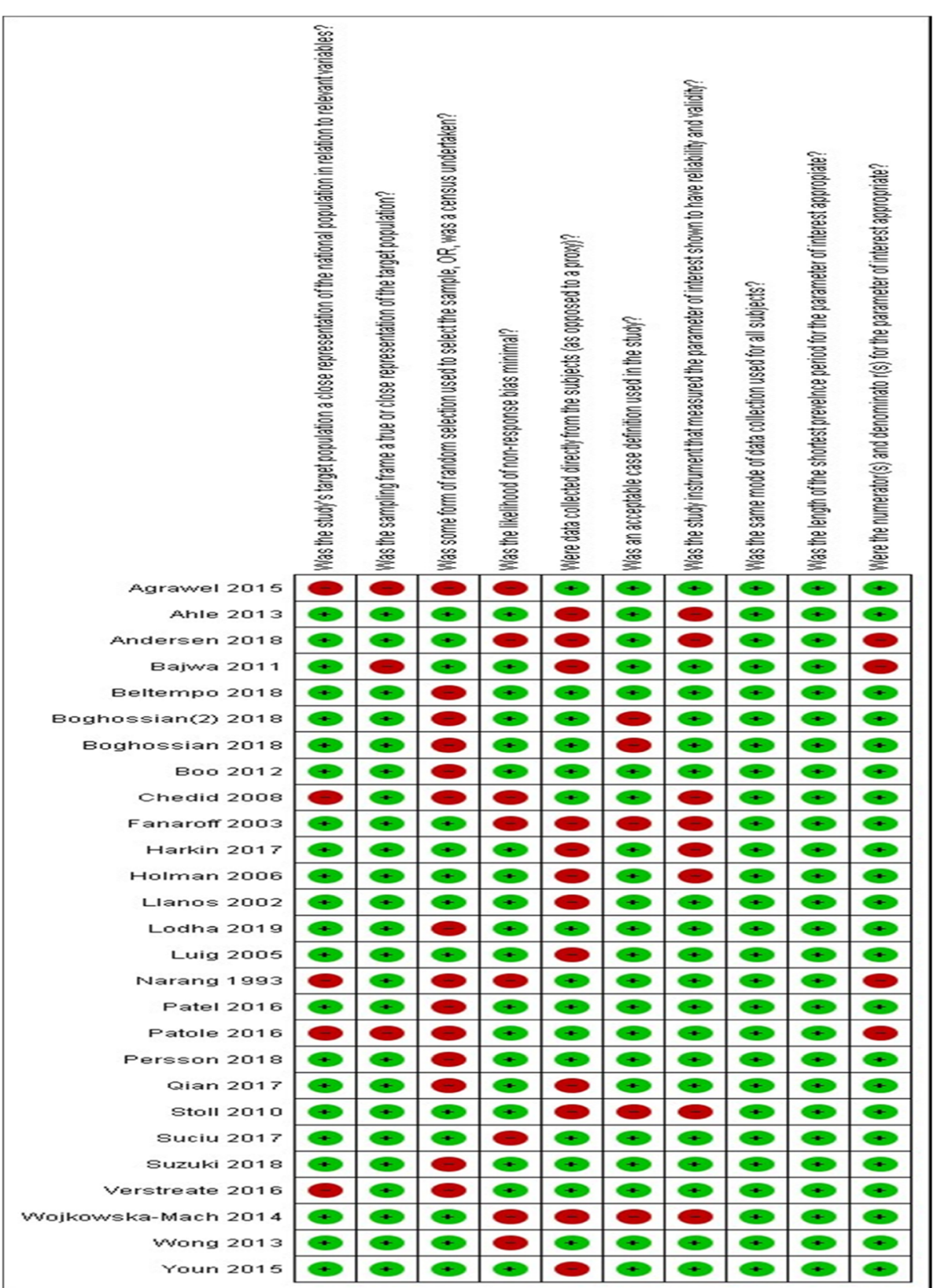

Fig. 3 Risk of bias plot that shows the methodological quality assessment of the 27 studies included 


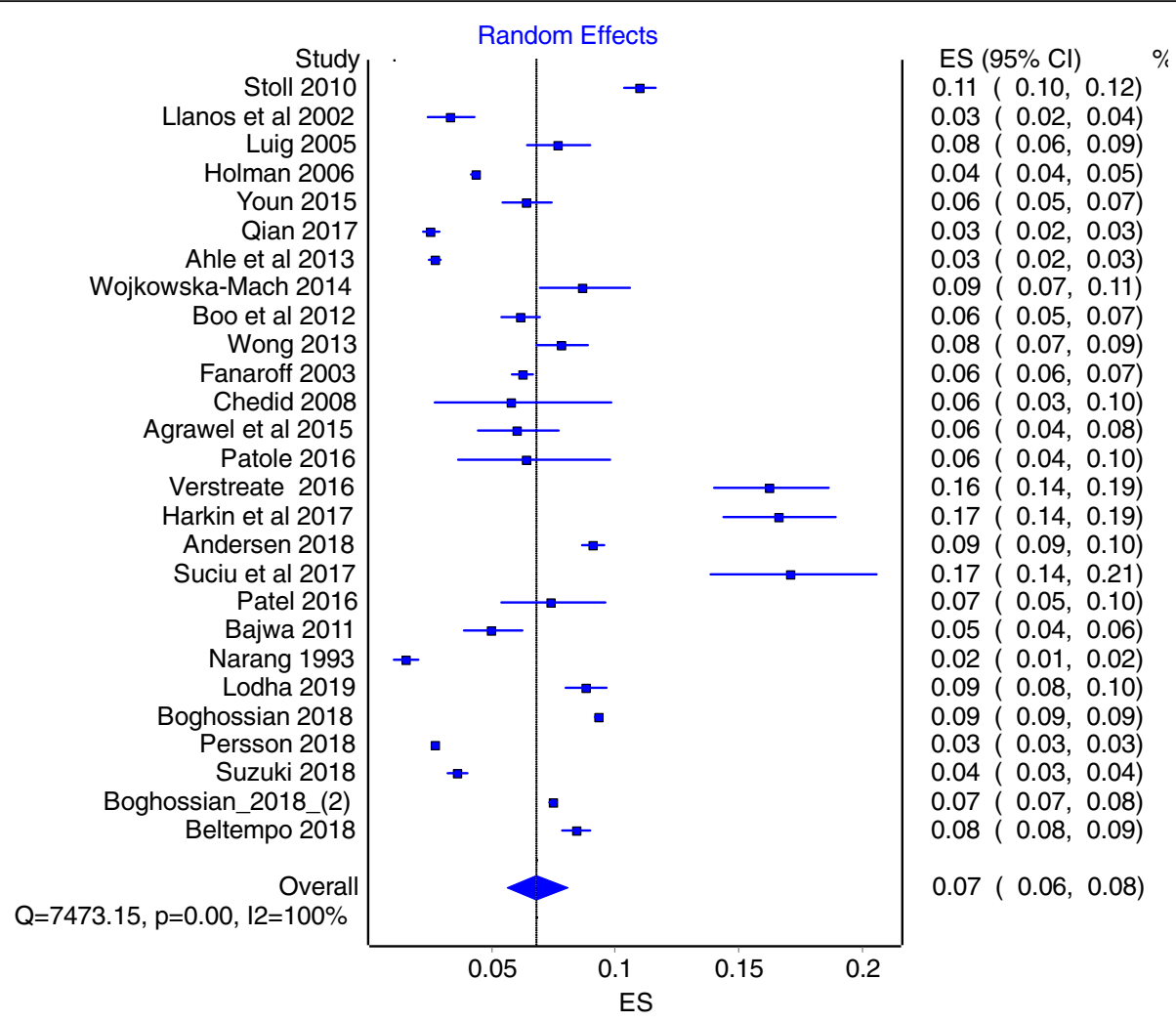

Fig. 4 Forrest plot obtained using Random Effect Model

Boghossaan et al. 2018, examined infants of gestational ages 22 to 29 weeks born between January 2006 and December 2016 at a Vermont Oxford Network center in the United States were. NEC developed in 18,129 among the 194,736 infants. The incidence of NEC was 9\% [26]. Persson et al. 2018, conducted a retrospective cohort study at 7 national networks in high-income countries that are part of the International Neonatal Network for Evaluating Outcomes in Neonates and used prospectively collected data on 76,360 very preterm, singleton infants. 2077 infants developed NEC and the incidence was $3 \%$ [27].

Suzuki et al. 2018, retrospectively examined 8245 extremely preterm infants born between 2008 and 2012 using Neonatal Research Network database in Japan. They estimated incidence to be $4 \%$ [28]. Boghossian et al. 2018, collected 138,869 large for gestational age infant's data from 852 US centers participating in the Vermont Oxford Network. The incidence of NEC was 7\% (10,376 new cases) [29]. Beltempo et al. 2018, collected data about extremely preterm infants born from 22 to 28 weeks' gestational age Canadian Neonatal Network. Study population was 9230 among them 778 developed NEC. The incidence of NEC was $8 \%$ [48].

\section{Assessment of risk of Bias}

The quality assessment of 27 individual studies carried out as per Hoy et al. [30] criteria are summarized graphically presented in Fig. 3. Studies performed very highly on components like use of consistent mode of data collection from all infants as well as sufficient follow up time required for the desired outcome to occur. However, only about $50 \%$ of the studies had a random selection of samples. Overall, most studies scored high and 17 out of 27 studies had a lower risk of bias based on a cut of $8 / 10$ or more as suggested by the Hoy's criteria.

\section{Quantitative analysis of incidence}

There were significant heterogeneity between studies, as indicated by $\mathrm{I}^{2}$ value of $100 \%$ and the Cochrane Q- statistics (value $=7473 ; P<0.0001$ ). As such we used REM as the main model to obtain our conclusions. REM estimate were $7.0 \%$ (95\% CI: 6.0-8.0\%) (Fig. 4), and additional quality adjusted QEM provided a sensitivity estimate of $6.0 \%$ (95\% CI: 4.0-9.0\%) (Fig. 5).

\section{Publication bias}

Hunter's modified funnel Plot [36] as appropriate for the incidence data used to evaluate the publication bias 


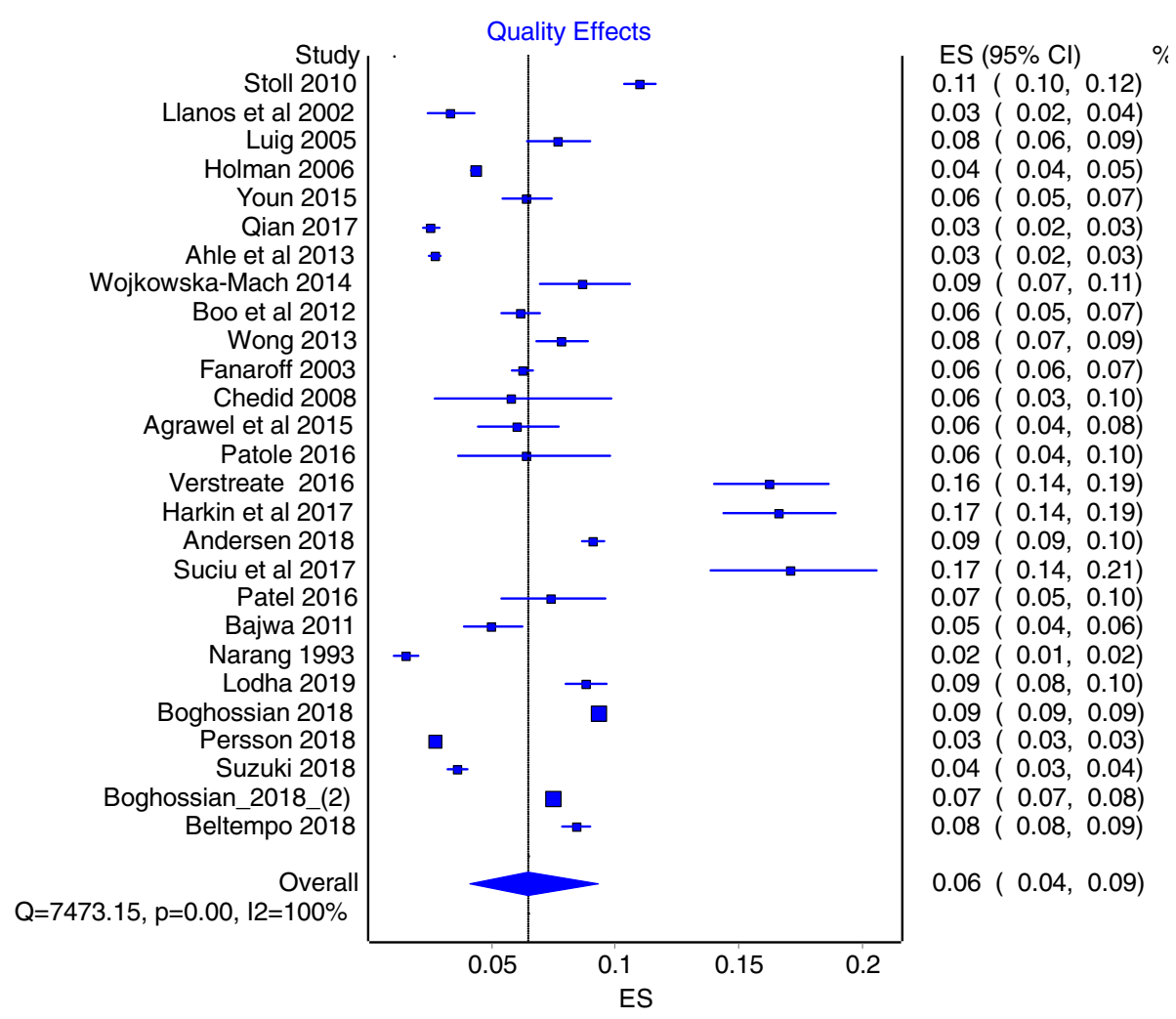

Fig. 5 Forrest plot obtained using Quality Effect Model

appear to not to show a serious concern (Fig. 6). Further, the Eggers regression confirmed that publication bias was not statistically significant (two tailed $p$-value $=$ 0.80 ). The Kendall's Tau test statistics was also not statistically indicating less likely that these studies encountered publication bias (two tailed $p$-value $=0.936$ ).

\section{Subgroup analysis}

There was no significant regional variation between North America, Western Europe and Australia as well as Asia (Table 3). There appear to be some variation between HIC and LMIC countries, although these differences were not statistically significant. No significant variation between VLBW infants and extreme prematurity was found.

\section{Meta-regression}

There was a statistically significant increase in the $\log$ event rate over time, quantified by the publication year (Fig. 7).

\section{Discussion}

This study is perhaps the first attempt to provide a pooled estimate of the incidence of Necrotizing
Enterocolitis in VLBW infants. Seven out of 100 of all VLBW infants in NICU are likely to develop NEC as per our synthesis. However, there were considerable heterogeneity in the estimates across studies. Such important variability may be driven by myriad of factors including the variation in the quality of health care systems.

Subgroup analysis based on geographic regions did not reveal any differences (i.e. South East Asia versus Europe, North America and Australia). However, when countries reporting the data on NEC were re-classified based on income levels using Word Bank classifications the incidence in high income counties (HIC) varied from the low and mid income countries (LMIC), although, these differences were not statistically significant. Such variation may be attributed to the fewer published studies from LMIC and potential under power to detect any differences. However, it is also possible that slightly lower incidence reported in LMIC may be due to higher gestational age cutoff point for resuscitation used in case of extreme prematurity. It is also possible that the sicker babies in LMIC may have had higher risk of mortality. As a result the population of neonates in LMIC may appear healthier and at lesser risk of developing NEC. 


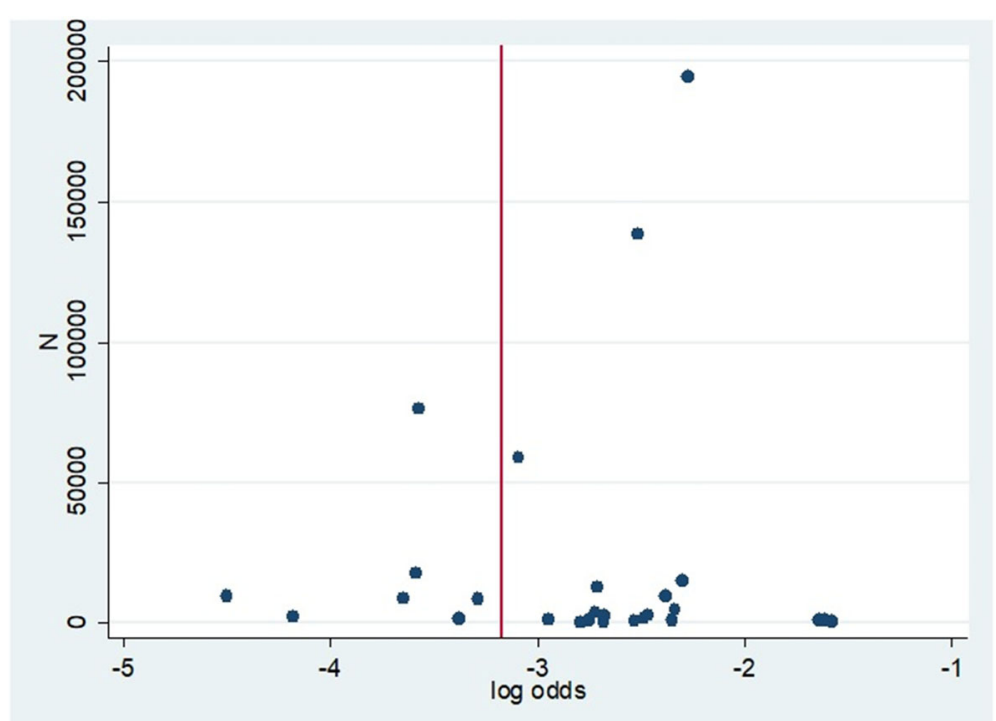

Fig. 6 Hunter's plot used to assess the publication bias

The increase in the incidence of NEC over time that our study found using meta-regression maybe attributed to multiple factors. Improvement in neonatal care and better survival of premature infants are possible causes as well as improvement in diagnosis and reporting. Increase in incidence of NEC over time can also be attributed to lack of wide scale prevention strategies. Ahle et al. demonstrated a jshaped distribution of incidence over time in Sweden. While the incidence was 150 per 10,000 live

Table 3 Subgroup analysis by region and income

\begin{tabular}{lll}
\hline Region & $\begin{array}{l}\text { Pooled Incidence } \\
(\%)\end{array}$ & $95 \% \mathrm{Cl}$ \\
\hline All & 6.0 & {$[4.0,9.0]$} \\
North America, Western Europe and & 4.3 & {$[2.5,6.6]$} \\
Australia & 3.9 & {$[1.4,7.3]$} \\
Asia & & \\
Income & 6.0 & {$[4.0,9.0]$} \\
$\quad$ All & 7.0 & {$[4.0,10.0]$} \\
$\quad$ High income countries (HIC) & 3.0 & {$[1.0,6.0]$} \\
$\quad$ Low and middle-income countries & & \\
$\quad$ (LMIC) & & {$[4.0,9.0]$} \\
Population at risk & 6.0 & {$[3.0,9.0]$} \\
$\quad$ All & 6.0 & {$[2.0,13.0]$} \\
$\quad$ VLBW infants & 7.0 & \\
$\quad$ Extremely premature & &
\end{tabular}

births among VLBW infants in the late 80 s, it increased to approximately 800 per 10,000 live births in VLBW, a multiple fold increase in later decade [12]. This increase may be related to variations in local health services. However, findings from the analysis of the NICHD data base from the United States reported [20] showed a different picture. They reviewed VLBW infants from three epochs: 19871988, 1993-1994, and 1999-2000. Their analysis compared the incidence across these three periods and they demonstrated that the incidence of NEC did not change over time. The data presented in our analysis represents a wider time period and a set of more diverse healthcare settings. Due to paucity of data available from lower income countries, the pooled estimate may have limited external validity and not fully generalizable to all global settings and populations.

Our findings, however, should be understood in the light of some limitations that this study encountered. Only 12 out 26 studies could be considered to be of higher quality and this may be linked to the substantially heterogeneity that we encountered. Although, we employed quality effect models to adjust for variation in study qualities, substantial heterogeneity noted in this study does pose a threat to evidence synthesis. The diagnosis of NEC using Bell's criteria or similar definitions schemes is a day to day clinical challenge. To a certain extent, two clinicians may justifiably disagree on labeling a baby as confirmed NEC versus suspected NEC. 


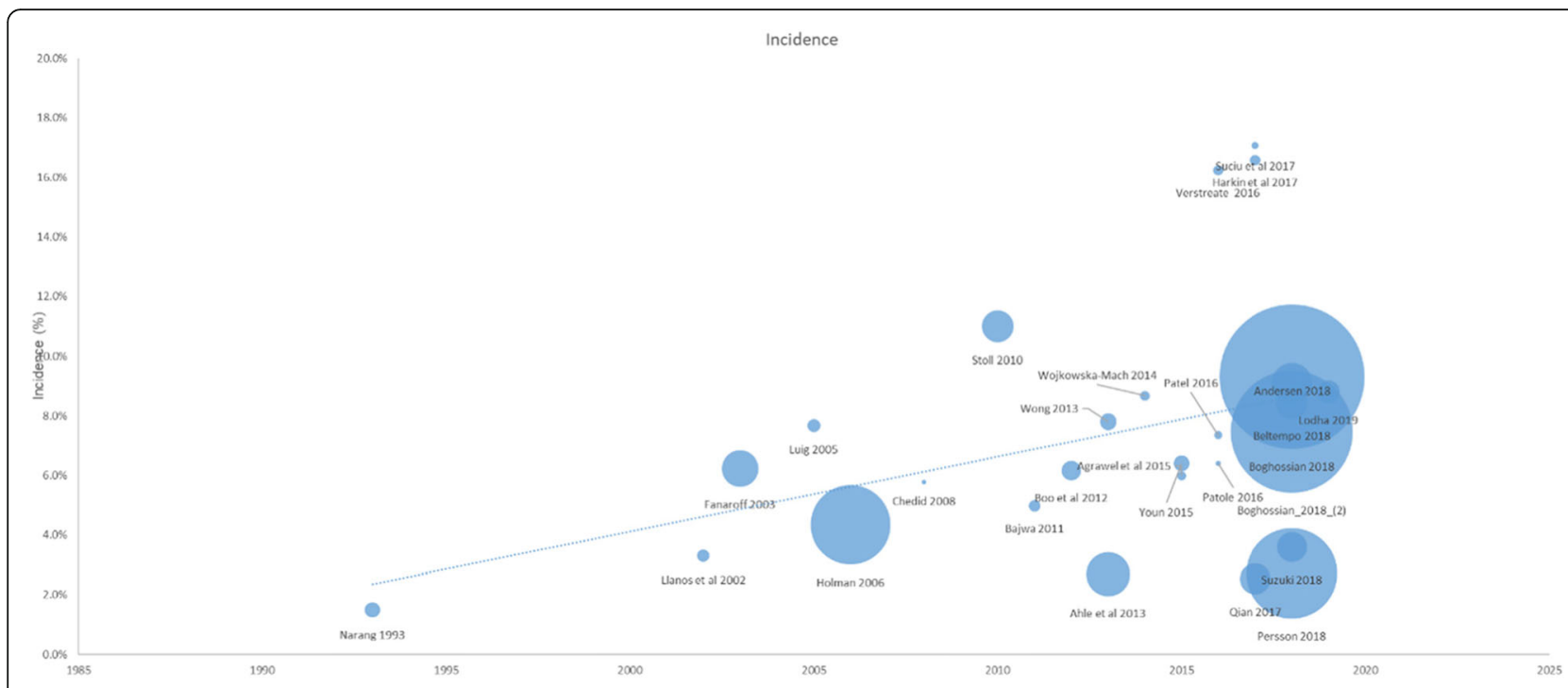

Fig. 7 Meta regression of incidence over time

\section{Conclusions}

Seven out of 100 infants admitted to NICU and are VLBW are likely to develop NEC. However, there are substantial variability in incidence reported from different parts of the world, likely be due to differences in clinical and health settings in addition to methodological variations. Larger and higher quality studies on incidence of NEC and associated factors, particularly form low and middle income countries are warranted.

\section{Supplementary information}

Supplementary information accompanies this paper at https://doi.org/10. 1186/s12887-020-02231-5.

\section{Additional file 1.}

\section{Abbreviations}

FEM: Fixed effect model; ICD: International classification of diseases; MeSH: Medical subject heading; NEC: Necrotizing enterocolitis; $\mathrm{NICH}$ D: National institute of child health and human development; PRIS MA: Preferred reporting items for systematic review and meta-analysis; QEM: Quality effect model; REM: Random effect model; RDS: Respiratory distress syndrome; VLBW: Very low birth weight

\section{Acknowledgements}

Not applicable.

\section{Authors' contributions}

AA and LT designed the study and developed the study protocol. AA did the searchers, data extraction and quality assessment under the supervision of LT. NI assisted AA in database search, data extraction and quality assessment. AA carried out the data synthesis and analyses. AA written the first draft of the manuscript that was critically revised by LT. All authors approved the final draft of the manuscript.

\section{Availability of data and materials}

Input data for the analyses are available from the corresponding author on request.

\section{Ethics approval and consent to participate}

Not applicable given this is a systematic review.

\section{Consent for publication}

Not applicable.

\section{Competing interests}

The authors declare that they have no competing interests.

\section{Author details}

'Department of Public Health, College of Health Sciences, QU Health, Qatar University, Doha, Qatar. ${ }^{2} \mathrm{HMC}$ Medical Cooperation, Doha, Qatar. ${ }^{3}$ Sidra Medicine, Doha, Qatar.

Received: 16 February 2020 Accepted: 30 June 2020

Published online: 13 July 2020

\section{References}

1. Thyoka M, Eaton S, Hall NJ, Drake D, Kiely E, Curry J, et al. Advanced necrotizing enterocolitis part 2: recurrence of necrotizing enterocolitis. Eur J Pediatr Surg. 2012;22(1):13-6 Available from: http://www.ncbi.nlm.nih.gov/ pubmed/22434228.

2. Guthrie SO, Gordon P V, Thomas V, Thorp JA, Peabody J, Clark RH. Necrotizing enterocolitis among neonates in the United States. J Perinatol. 2003;23(4):278-285. Available from: http://eutils.ncbi.nlm.nih.gov/entrez/ eutils/elink.fcgi?dbfrom=pubmed\&id=12774133\&retmode $=$ ref\& $\mathrm{cmd}=$ prlinks\%5Cnpapers3://publication/doi/https://doi.org/10.1038/sj.jp.7210892.

3. Llanos AR, Moss ME, Pinzòn MC, Dye T, Sinkin RA, Kendig JW. Epidemiology of neonatal necrotising enterocolitis: a population-based study. Paediatr Perinat Epidemiol. 2002;16(4):342-9 Available from: http://www.ncbinlm.nih. gov/pubmed/12445151.

4. Luig M, Lui K. NSW \& ACT NICUS Group. Epidemiology of necrotizing enterocolitis--Part I: Changing regional trends in extremely preterm infants over 14 years. J Paediatr Child Health. 2005;41(4):169-73 Available from: http://www.ncbi.nlm.nih.gov/pubmed/15813869

5. Luig M, Lui K. NSW \& ACT NICUS Group. Epidemiology of necrotizing enterocolitis--Part II: Risks and susceptibility of premature infants during the surfactant era: a regional study. J Paediatr Child Health. 2005:41(4):174-9 Available from: http://www.ncbi.nlm.nih.gov/pubmed/15813870.

6. Thompson A, Bizzarro M, Yu S, Diefenbach K, Simpson BJ, Moss RL, Risk factors for necrotizing enterocolitis totalis: a case-control study. J Perinatol

\section{Funding}

None declared. 
2011;31(11):730-738. Available from: http://www.nature.com/doifinder/ https://doi.org/10.1038/jp.2011.18

7. Chedid F, Shanteer S, Haddad H, Musharraf I, Shihab Z, Imran A, et al. Shortterm outcome of very low birth weight infants in a developing country: comparison with the Vermont Oxford network. J Trop Pediatr. 2009:55(1):15-9.

8. Goldman HI. Feeding and necrotizing enterocolitis. Am J Dis Child. 1980; 134(6):553-5 Available from: http://www.ncbi.nlm.nih.gov/pubmed/6770672.

9. Kliegman RM, Fanaroff AA. Neonatal necrotizing enterocolitis: a nine-year experience. Am J Dis Child. 1981;135(7):603-7 Available from: http://www. ncbi.nlm.nih.gov/pubmed/6787912.

10. Gaynes RP, Palmer S, Martone WJ, Holt CL, Buchter DS, Frawley LW, et al. The role of host factors in an outbreak of necrotizing enterocolitis. Am J Dis Child. 1984; 138(12):1118-20 Available from: http://www.ncbi.nlm.nih.gov/pubmed/6507393.

11. Wilson R, Kanto WP, McCarthy BJ, Burton T, Lewin P, Terry J, et al. Epidemiologic characteristics of necrotizing enterocolitis: a populationbased study. Am J Epidemiol. 1981;114(6):880-7 Available from: http://www. ncbi.nlm.nih.gov/pubmed/7315835.

12. Ahle $M$, Drott $P$, Andersson RE. Epidemiology and trends of necrotizing Enterocolitis in Sweden: 1987-2009. Pediatrics 2013;132(2):e443-e451. Available from: http://pediatrics.aappublications.org/cgi/doi/https://doi.org/ 10.1542/peds.2012-3847.

13. Wójkowska-Mach J, Rózańska A, Borszewska-Kornacka M, Domańska J, Gadzinowski J, Gulczyńska E, et al. Necrotising enterocolitis in preterm infants: Epidemiology and antibiotic consumption in the Polish neonatology network neonatal intensive care units in 2009. PLoS One. 2014;9(3):e92865.

14. Boo NY, Cheah IG. Risk factors associated with necrotising enterocolitis in very low birth weight infants in Malaysian neonatal intensive care units. Singapore Med J. 2012;53(12):826-31 Available from: http://www.ncbi.nlm. nih.gov/pubmed/23268157.

15. Lu Q, Cheng S, Zhou M, Yu J. Risk factors for necrotizing Enterocolitis in neonates: A retrospective case-control study. Pediatr Neonatol. 2017;58(2):165-70.

16. Youn YA, Kim EK, Kim SY. Necrotizing enterocolitis among very-low-birthweight infants in Korea. J Korean Med Sci. 2015;30:S75-80.

17. Moher D, Liberati A, Tetzlaff J, Altman DG. Preferred reporting items for systematic reviews and meta-analyses: the PRISMA statement. J Clin Epidemiol. 2009;62(10):1006-12.

18. Stoll BJ, Hansen NI, Bell EF, Shankaran S, Laptook AR, Walsh MC, et al. Neonatal outcomes of extremely preterm infants from the NICHD. Pediatrics. 2010;126(3):443-56.

19. Holman RC, Stoll BJ, Curns AT, Yorita KL, Steiner CA, Schonberger LB. Necrotising enterocolitis hospitalisations among neonates in the United States. Paediatr Perinat Epidemiol. 2006;20(6):498-506.

20. Fanaroff AA, Hack M, Walsh MC. The NICHD Neonatal research network: changes in practice and outcomes during the first 15 years. Semin Perinatol. 2003;27(4):281-7.

21. Patole SK, Rao SC, Keil AD, Nathan EA, Doherty DA, Simmer KN. Benefits of bifidobacterium breve M-16V supplementation in preterm neonates -A retrospective cohort study. PLoS One. 2016;11(3):1-11.

22. Suciu LM, Puscasiu L, Cucerea M, Szabo B, Ognean ML, Petrescu O, et al. Trends in outcomes of very preterm infants in Romania: A tale of three cities. Pediatr Int. 2017;59(11):1157-64.

23. Bajwa NM, Berner M, Worley S, Pfister RE, Zeilinger G, Bührer C, et al. Population-based age stratified morbidities of premature infants in Switzerland. Swiss Med Wkly. 2011;141(JUNE):1-6.

24. Narang A, Rao R, Bhakoo ON. Neonatal necrotizing enterocolitis an epidemiological study. Indian Pediatr. 1993;30(10):1207-14 Available from: http://www.ncbi.nlm.nih.gov/pubmed/8077012.

25. Lodha A, Shah PS, Soraisham AS, Rabi Y, Abou Mehrem A, Singhal N. Association of Deferred vs immediate cord clamping with severe neurological injury and survival in extremely low-gestational-age neonates. JAMA Netw Open. 2019;2(3):e191286.

26. Boghossian NS, Geraci M, Edwards EM, Horbar JD. In-hospital outcomes in large for gestational age infants at 22-29 weeks of Gestation. J Pediatr. 2018;198:174-180.e13.

27. Persson M, Shah PS, Rusconi F, Reichman B, Modi N, Kusuda S, et al. Association of maternal diabetes with Neonatal outcomes of very preterm and very low-birth-weight infants an international cohort study. JAMA Pediatr. 2018;172(9):867-75

28. Suzuki Y, Kono Y, Hayakawa T, Shimozawa H, Matano M, Yada Y. Neonatal factors related to center variation in the incidence of late-onset circulatory collapse in extremely preterm infants. PLoS One. 2018;13(6):e0198518.
29. Boghossian NS, Geraci M, Edwards EM, Horbar JD. Sex differences in mortality and morbidity of infants born at less than 30 weeks' gestation. Pediatrics. 2018;142(6):e20182352.

30. Hoy D, Brooks P, Woolf A, Blyth F, March L, Bain C, et al. Assessing risk of bias in prevalence studies: modification of an existing tool and evidence of interrater agreement. J Clin Epidemiol 2012;65(9):934-939. Available from: https://doi.org/10.1016/j.jclinepi.2011.11.014.

31. Barendregt JJ, Doi SA, Lee YY, Norman RE, Vos T. Meta-analysis of prevalence. J Epidemiol Community Health. 2013;67(11):974-8.

32. Higgins JPT, Thompson SG. Quantifying heterogeneity in a meta-analysis. Stat Med. 2002;21(11):1539-58 Available from: http://www.ncbi.nlm.nih.gov/ pubmed/12111919.

33. Joanna Briggs Institute: The systematic review of prevalence and incidence data. Joanna Briggs Inst Rev Man 2014. 2014;South Aust.

34. Higgins JPT, Green S (editors). Cochrane Handbook for Systematic Reviews of Interventions Version 5.1.0 [updated March 2011]. The Cochrane Collaboration, 2011. Available from https://www.handbook.cochrane.org.

35. Doi SAR, Thalib L. A quality-effects model for meta-analysis. Epidemiology. 2008; 19(1):94-100 Available from: http://www.ncbi.nlm.nih.gov/pubmed/18090860.

36. Hunter JP, Saratzis A, Sutton AJ, Boucher RH, Sayers RD, Bown MJ. In metaanalyses of proportion studies, funnel plots were found to be an inaccurate method of assessing publication bias. J Clin Epidemiol. 2014;67(8):897-903.

37. Fantom NJ, Serajuddin U. The World Bank's classification of countries by income (English). Policy Research working paper; no. WPS 7528. Washington, D.C.: World Bank Group. 2016. http://documents.worldbank. org/curated/en/408581467988942234/The-World-Banks-classification-ofcountries-by-income.

38. Michael Borenstein. Comprehensive Meta Analyses (CMA) 3.3.070. In: CMA, biostat, New Jersey, USA. New Jersey: Biostat; 2014.

39. Anderson JG, Rogers EE, Baer RJ, Oltman SP, Paynter R, Partridge JC, et al. Racial and ethnic disparities in preterm infant mortality and severe morbidity: A population-based study. Neonatology. 2017:44-54.

40. Motta C, Scott W, Mahony L, Koch J, Wyckoff M, Reisch J, et al. The association of congenital heart disease with necrotizing enterocolitis in preterm infants: A birth cohort study. J Perinatol. 2015;35(11):949-53.

41. Patel RM, Denning PW. Intestinal microbiota and its relationship with necrotizing enterocolitis. Pediatr Res. 2015;78(3):232-8 Available from: http:// www.ncbi.nlm.nih.gov/pubmed/25992911.

42. Verstraete EH, Mahieu L, De Coen K, Vogelaers D, Blot S. Impact of healthcare-associated sepsis on mortality in critically ill infants. Eur J Pediatr [Internet] 2016;175(7):943-952. Available from: http://dx.doi.org/https://doi. org/10.1007/s00431-016-2726-6.

43. Härkin P, Marttila R, Pokka T, Saarela T, Hallman M. Morbidities associated with patent ductus arteriosus in preterm infants. Nationwide cohort study. J Matern Neonatal Med. 2017;0(0):1-8. Available from: https://www. tandfonline.com/doi/full/https://doi.org/10.1080/14767058.2017.1347921.

44. Qian T, Zhang R, Zhu L, Shi P, Yang J, Yang C, et al. Necrotizing enterocolitis in low birth weight infants in China: Mortality risk factors expressed by birth weight categories. Pediatr Neonatol. 2016;1-7. Available from: http://dx.doi. org/https://doi.org/10.1016/j.pedneo.2016.10.004.

45. Agarwal P, Sriram B, Rajadurai VS. Neonatal outcome of extremely preterm Asian infants $\leq 28$ weeks over a decade in the new millennium. J Perinatol 2015;35(4): 297-303. Available from: http://dx.doi.org/https://doi.org/10.1038/jp.2014.205.

46. Wong D, Abdel-Latif ME, Kent AL. Antenatal steroid exposure and outcomes of very premature infants: A regional cohort study. Arch Dis Child Fetal Neonatal Ed. 2014;99(1).

47. Garey CL, Laituri CA, Little DC, Ostlie DJ, St Peter SD. Outcomes of perforated appendicitis in obese and nonobese children. J Pediatr Surg. 2011;46(12):2346-8 Available from: http://www.ncbi.nlm.nih.gov/ pubmed/22152879.

48. Beltempo M, Shah PS, Ye XY, Afifi J, Lee S, McMillan DD. SNAP-\|l for prediction of mortality and morbidity in extremely preterm infants. J Matern Neonatal Med. 2019;32(16):2694-2701. Available from: https://doi.org/ https://doi.org/10.1080/14767058.2018.1446079.

\section{Publisher's Note}

Springer Nature remains neutral with regard to jurisdictional claims in published maps and institutional affiliations. 